\title{
High-resolution Analytical STEM of Defects and Interfaces in Beam-sensitive Ultra- thin Cuprate Films
}

\author{
Vesna Srot ${ }^{1}$, Yi Wang ${ }^{1}$, Matteo Minola ${ }^{1}$, Ute Salzberger ${ }^{1}$, Marco Salluzzo ${ }^{2}$, Gabriella Maria De Luca ${ }^{3}$, \\ Bernhard Keimer $^{1}$ and Peter van Aken ${ }^{1}$ \\ ${ }^{1}$ Max Planck Institute for Solid State Research, Stuttgart, Baden-Wurttemberg, Germany, ${ }^{2}$ CNR-SPIN, \\ Napoli, Campania, Italy, ${ }^{3}$ Dipartimento di Fisica “'E. Pancini”, Napoli, Campania, Italy
}

In functional complex oxides, exciting phenomena emerging at the atomic scale have been identified due to major technological as well as methodological development in scanning transmission electron microscopy (STEM). Simultaneous acquisition of annular bright-field (ABF-) and high-angle annular dark-field (HAADF-) STEM images in combination with advanced analytical methods, such as atomiccolumn-resolved electron energy-loss spectroscopy (EELS), sets a remarkable approach in the study of thin films.

In the present research, $\mathrm{NbBa}_{2} \mathrm{Cu}_{3} \mathrm{O}_{7}$ (NBCO) ultra-thin films with a thickness of 4 unit cells have been deposited on $\mathrm{TiO}_{2}$-terminated $\mathrm{SrTiO}_{3}$ (STO) substrate by high oxygen pressure diode sputtering [1]. The stacking sequence of NBCO layers along the crystallographic $c$-axis is as follows: $\mathrm{BaO}-\mathrm{CuO}_{2}-\mathrm{Nd}_{-}-\mathrm{CuO}_{2}-$ $\mathrm{BaO}-\mathrm{CuO}$. In perovskite-type-structure layered $\mathrm{NBCO}$, the $\mathrm{Cu}$ atoms are located in the $\mathrm{CuO}_{2}$ planes as well as in the $\mathrm{CuO}$ chains that run parallel to the planes. $\mathrm{Nd}$ atoms are situated in-between two $\mathrm{CuO}_{2}$ planes, while $\mathrm{Ba}$ atoms are present between the planes and the chains. Oxygen content fluctuations can cause major changes to the physical properties [2], and the charge balance between the $\mathrm{CuO}_{2}$ planes and $\mathrm{CuO}$ chains in NBCO strongly affects the $T c[3]$.

Electron transparent TEM lamellae of a NBCO/STO sample were prepared in cross-sectional orientation using two different approaches [4]: (i) Combination of tripod (wedge-shaped) polishing followed by $\mathrm{Ar}^{+}$ ion-milling, while cooling the sample with L-N2, and (ii) preparation of TEM lamellae with a focused ion beam (FIB) (Zeiss CrossBeam XB 1540) followed by additional thinning and polishing in a Nanomill system (Fischione Model 1040).

We have combined atomically resolved quantitative STEM imaging and analytical techniques to investigate the ultra-thin NBCO films in an advanced aberration-corrected JEOL JEM-ARM200F microscope equipped with a DCOR probe corrector and GIF Quantum K2 system operated at $200 \mathrm{kV}$. Simultaneously recorded and superimposed HAADF- and ABF-STEM images of an area with visible defects in NBCO and surface step on STO (left) and an ABF-STEM image with the overlaid NBCO structural model (right) are presented in Figure 1. A multiple frame acquisition routine [5] enabled the improvement of the signal-to-noise ratio as well as the reduction of the image distortions.

Atomically resolved ADF-STEM imaging combined with EELS spectrum mapping was applied to investigate the chemical identity of ultra-thin NBCO films, their defects and the NBCO/STO interfaces. An EELS elemental map from an area, where defects in the NBCO film and a surface step on STO substrate are clearly visible, is shown in Figure 2. We have confirmed that the STO substrate is $\mathrm{TiO}_{2}$ terminated ([6] and left side of the map). Furthermore, we have detected a double $\mathrm{TiO}_{2}$ layer at the 
interface region in certain areas of the sample, where surface steps appear (right side of the map). Consequently, such areas always show the presence of defects within NBCO films and possible mechanisms governing the formation of such defect will be discussed. [7]

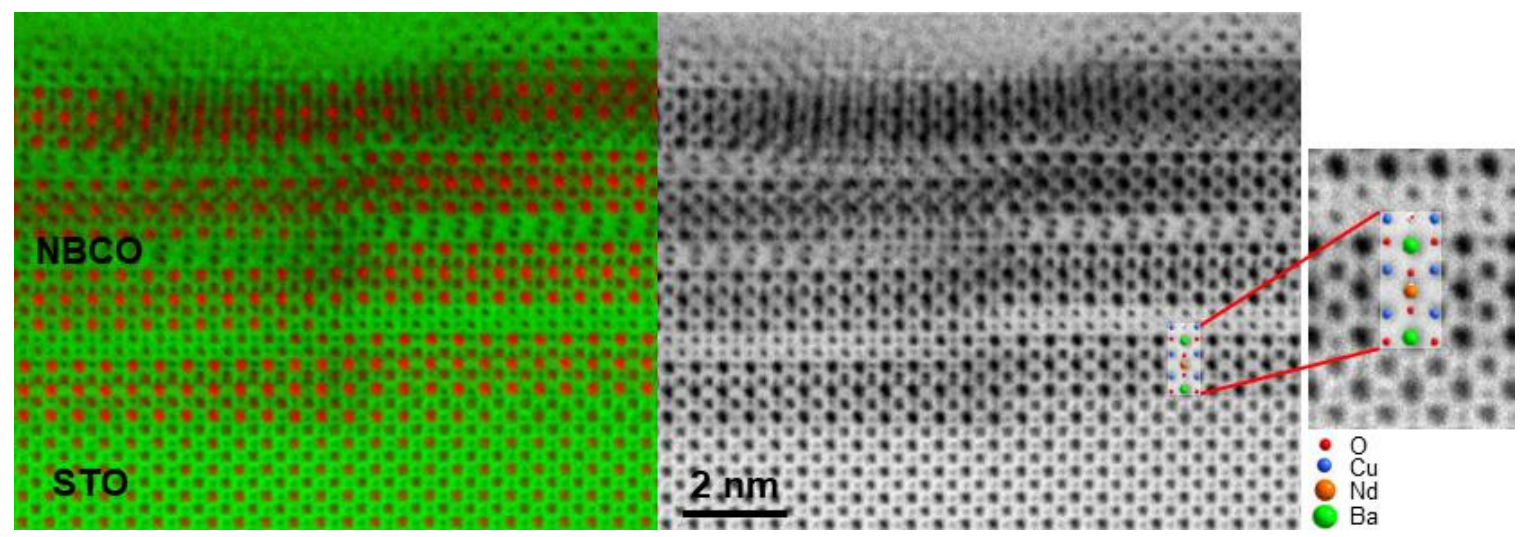

Figure 1. Superimposed simultaneously acquired HAADF- (red) and ABF- (green) STEM images (left) of an epitaxial NBCO ultra-thin film (4 unit cells thick) grown on STO and ABF-STEM image (right) with an overlay of the NBCO structural model. Please note the presence of a defect due to a surface step on the STO substrate.

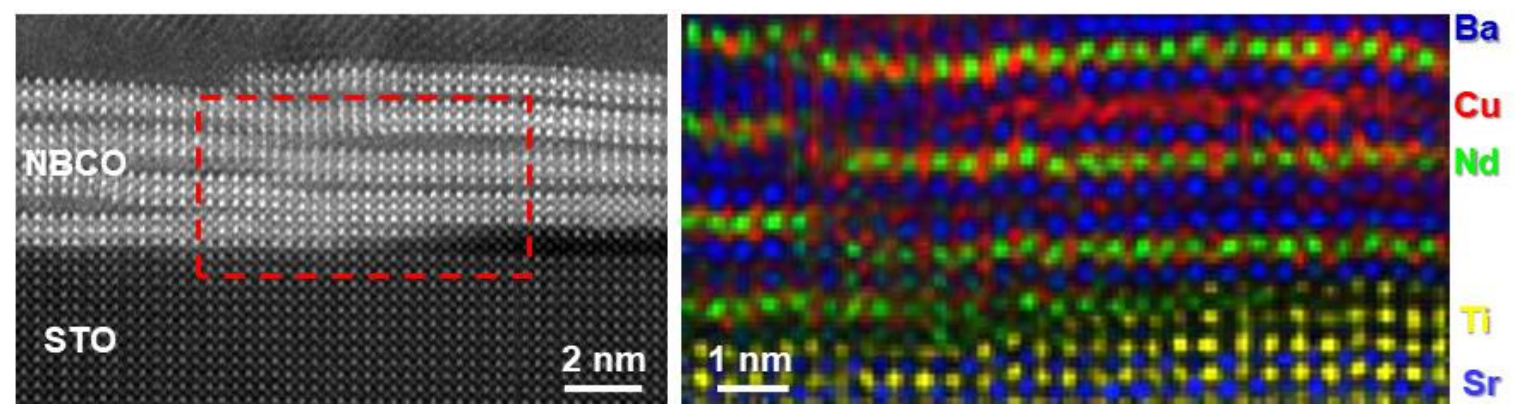

Figure 2. ADF-STEM image of the NBCO/STO interface with a corresponding atomically resolved EELS elemental map obtained from the area, where defects in NBCO and a surface step on STO are clearly visible.

\section{References}

[1] M Salluzzo et al. Phys rev B 78 (2008) 054524.

[2] H Shaked et al., Phys Rev B 41 (1990) 4173.

[3] RJ Cava et al., Physica C 165 (1990) 419.

[4] V Srot et al., Microsc Microanal 25 (Suppl 2) (2019) 686.

[5] Y Wang et al., Ultramicroscopy 168 (2016) 46.

[6] V Srot et al., Microsc Microanal 25 (Suppl 2) (2019) 1750.

[7] This project has received funding from the European Union's Horizon 2020 research and innovation programme under grant agreement No. 823717 - ESTEEM3. 